\title{
Erbium Doped Fiber Lasers for Long Distance Communication Using Network of Fiber Optics
}

\author{
Thalangunam Krishnaswamy Subramaniam \\ Department of Science \& Humanities (Physics), Sri Sairam Engineering College, Chennai, India
}

Email address:

subramaniam.phy@sairam.edu.in

\section{To cite this article:}

Thalangunam Krishnaswamy Subramaniam. Erbium Doped Fiber Lasers for Long Distance Communication Using Network of Fiber Optics. American Journal of Optics and Photonics. Vol. 3, No. 3, 2015, pp. 34-37. doi: 10.11648/j.ajop.20150303.11

\begin{abstract}
A fiber doped with erbium laser is used in long distance communication over networks of fiber optics. Especially, in under water communication links where optical signals can be amplified by joining erbium-doped fiber at appropriate locations in the network of the fiber. Thus, a signal, when optically transmitted will get amplified and this will be useful when the signal travels between continents. There is no need for converting an optical signal into an electrical signal, amplified and then reintroduced into the fiber for transmission. The latest research trend is to produce ultra-short pulse widths of the order of $100 \mathrm{fs}$ and with a pulse repetition rate of the order of $10 \mathrm{GHz}$. Here I have used an $\mathrm{Er}^{3+}$ doped silica fiber for optical pumping $\lambda=0.98 \mu \mathrm{m}$ or $\lambda=1.48 \mu \mathrm{m}$ wavelength using radiation from a diode laser [Fig. 1]. A coupler was used to select the wavelength, which permits this light to reach the optical fiber transmission system, without disturbing the signal. The optical fiber is spliced into the transmission lines, which acts as an optical isolator. This fiber is constantly pumped by the diode laser. When the pulses pass through the system, they get amplified, while the pump light is reduced.
\end{abstract}

Keywords: Fiber Optics, Erbium-Doped, Spliced, Pulse Repetition Rate, Optical Isolator

\section{Introduction}

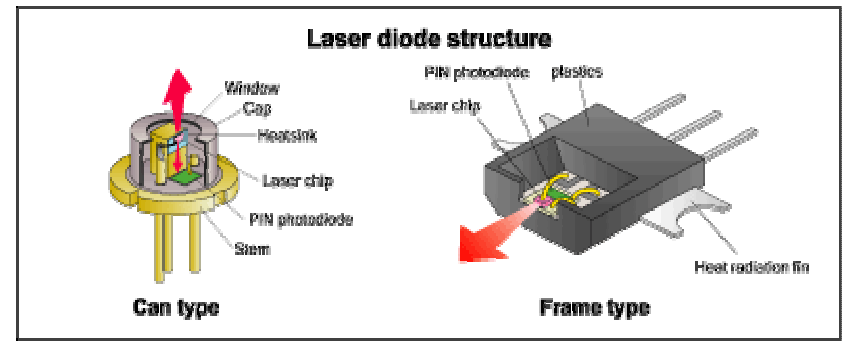

Figure 1. Diode laser structure used as a pump beam (Courtesy: Wikipedia).

Many rare-earth atoms like Erbium, etc, can be employed as dopants in crystals or in glass. When external sources are used, these active elements can be pumped to reach the desired level of 'population inversion'. In fiber optic communications, normally one is interested in wavelengths between $\lambda=1540$ to $1550 \mathrm{~nm}$ region and Erbium doped silica fibers are suitable for this purpose. The same fibers when pumped with $\lambda=0.98 \mu \mathrm{m}$ or $\lambda=1.48 \mu \mathrm{m}$ are used as optical amplifiers in optical communication systems [Ref 14]. In order to convert its application from an amplifier to an oscillator, Erbium doped with silica fiber is placed in an 'optical resonator', by using Fiber Bragg gratings (FBG) [Fig.2] below.

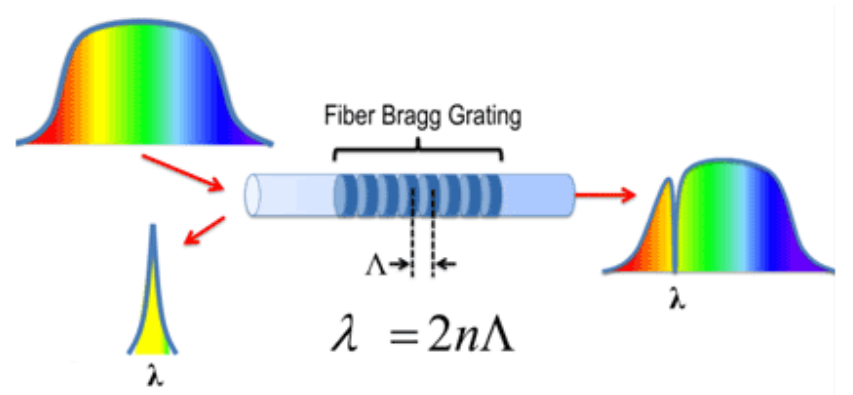

Figure 2. Fiber Bragg Grating (Courtesy: Wikipedia).

\section{Fiber Bragg Grating}

The fiber Bragg grating is used for an all fiber wavelength demultiplexer.A Germanium doped silica glass is photosensitive and when ultraviolet (UV) light is passed through the fiber; the refractive index of the doped silica glass is slightly changed. It is thus possible to create a periodic variation in the refractive index of the core itself, thereby creating a 'phase grating'. Using the Bragg's 
condition, that

$$
\lambda_{\mathrm{B}}=2 \Lambda \mathrm{n}_{\mathrm{eff}}
$$

where $\lambda_{B}$ is the Bragg wavelength, $\Lambda$ is the 'grating period', $\mathrm{n}_{\text {eff }}$ is the effective index of the mode; large coupling may arise between to and fro propagating modes. With a proper design, one can ensure that most of the power is reflected effectively and other signals are transmitted. Thus, the main use of such a FBG is that they are fiber compatible and the loss generated due to interconnection between fibers is very low.

At both the ends of the fiber, FBG act as mirrors in a laser resonator [Fig.3].

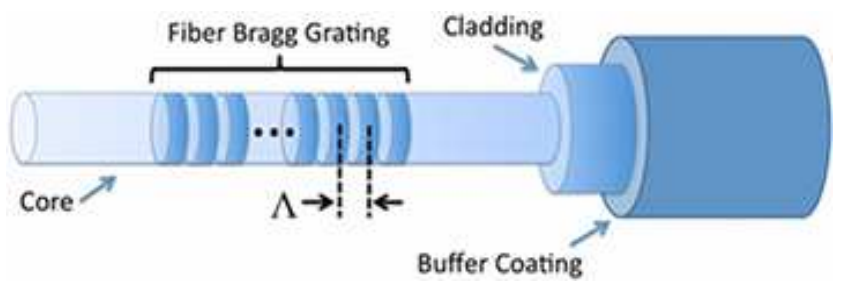

Figure 3. Fiber Bragg Grating acts as mirrors on both sides (Courtesy: Wikipedia).

\section{Energy Levels and Mechanism of Excitation in Optical Fiber Laser}

\subsection{Energy Levels}

The Erbium doped silica fiber laser is a three-level laser. The energy level scheme for the optical pumping is shown below here [Fig.4].

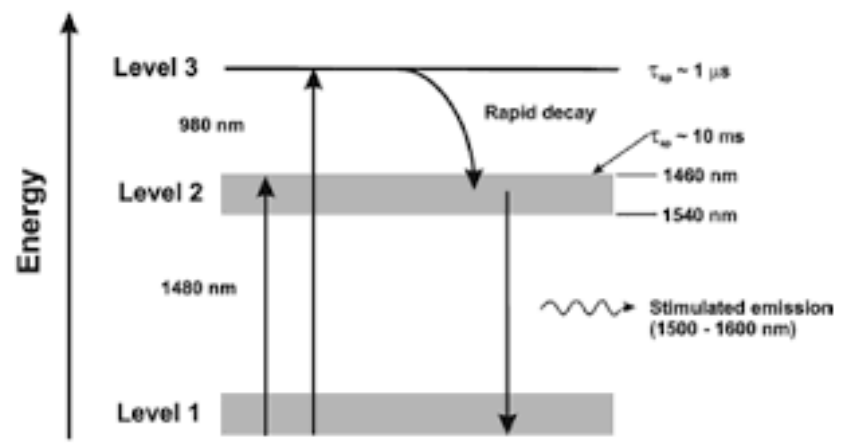

(The energy levels of $\mathrm{Er}^{3+}$ ion shows two absorption peaks at $0.98 \mu \mathrm{m}$ and at $1.48 \mu \mathrm{m}$.Here, on the Y-axis the energy is plotted in wave numbers and $1 \mathrm{~cm}^{-1}$ $\equiv v=30 \mathrm{GHz}$.)

Figure 4. Energy level diagram of erbium doped fiber laser (Courtesy Wikipedia).

In this scheme, optical pumping takes place from the ground state ${ }^{4} \mathrm{I}_{15 / 2}$ to the ${ }^{4} \mathrm{I}_{11 / 2}$ at a wavelength of $\lambda=$ $0.98 \mu \mathrm{m}$.Optical pumping can also occur from the lowest levels (ground state) to the upper laser state, ${ }^{4} \mathrm{I}_{13 / 2}$ at $\lambda=1.48 \mu \mathrm{m}$. Then rapid relaxation occurs to lowest sub-levels of ${ }^{4} \mathrm{I}_{13 / 2}$ from which 'laser action' is obtained in the region from $\lambda=1.53 \mu \mathrm{m}$ to $\lambda=1.56 \mu \mathrm{m}$. The mechanism of 'population inversion' is strange in this level. The lower level of the fiber optic laser acts similar to a dye laser. Higher levels of this state are not populated to a significant extent and hence higher levels act as lower level of population inversion. Thus there will be a non-radiative decay of population into the lower levels of ${ }^{4} \mathrm{I}_{13 / 2}$ state [Ref 5-7].

\subsection{Mechanism of Lasing}

The Erbium $\left(\mathrm{Er}^{3+}\right)$ is a rare-earth ion and has eleven electrons in the $4 \mathrm{f}$ orbital. It has a wide 'gain bandwidth' resulting from transition between the ${ }^{4} \mathrm{I}_{15 / 2}$ and the ${ }^{4} \mathrm{I}_{13 / 2}$ states. When this fiber is placed in a host medium all the $(2 \mathrm{~J}+1)$ components of the multiplets of this erbium ion are split and are homogeneously broadened due to neighboring oxygen ions in the silica glass. Hence all the sub-levels are further broadened due to inhomogeneous broadening, resulting in a wide "gain bandwidth" which is useful for the broadband optical communication application. The absorption crosssection of the levels, ${ }^{4} \mathrm{I}_{15 / 2} \rightarrow{ }^{4} \mathrm{I}_{11 / 2}(\lambda=1.48 \mu \mathrm{m})$ and the levels ${ }^{4} \mathrm{I}_{15 / 2} \rightarrow{ }^{4} \mathrm{I}_{13 / 2}(\lambda=0.98 \mu \mathrm{m})$ give rise to two strong peaks. The ${ }^{4} \mathrm{I}_{15 / 2}$ level is split into sixteen sub-levels $(2 \mathrm{~J}+1$, with $\mathrm{J}=15 / 2)$ and the ${ }^{4} \mathrm{I}_{13 / 2}$ level is split into fourteen sub-levels $(2 \mathrm{~J}+1$, with $\mathrm{J}=13 / 2$ ).Population inversion can be obtained through optical pumping using the $\lambda=0.98 \mu \mathrm{m}$, which lifts the electrons to the upper state ${ }^{4} \mathrm{I}_{11 / 2}$. An electron at this state has a $1 \mu \mathrm{sec}$ as lifetime and then it decays to the intermediate state ${ }^{4} \mathrm{I}_{11 / 2}$. The other intermediate state ${ }^{4} \mathrm{I}_{13 / 2}$ is a metastable state and has a lifetime of $\mathrm{t}=10^{-3}$ seconds. Each decay results in spontaneous emission of a photon and so $\mathrm{t}=\mathrm{t}_{\text {spontaneous. }}$. It may be noted here, that it is because of this long duration of 'spontaneous lifetime', lack of "Crosstalk" between communication channels have been made possible. Hence Erbium silica fiber is a three level laser and the laser emission uses the ground state as the lowest state. It should be noted that the erbium doped glass fiber laser must be pumped hard in order to achieve gain [Fig.5].

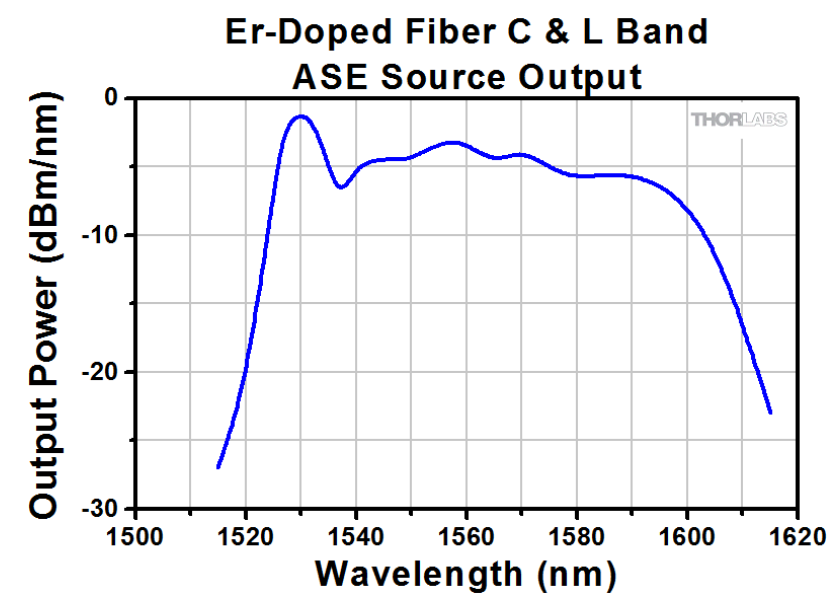

Figure 5. Wavelength versus output power in erbium doped fiber laser (Courtesy: Wikipedia).

Also, without a Fiber Bragg Grating (FBG), the erbium fiber can be used only as an inline optical amplifier in telecommunication networks. The following Table I below 
gives the properties of an Erbium-doped fiber used as an amplifier.

Table 1. Properties of erbium fiber amplifiers (Courtesy: Oxford University Press).

\begin{tabular}{ll}
\hline Properties of Amplifiers & Erbium doped fiber amplifiers \\
Active Medium & $\mathrm{Er}^{3+}$ ion in silica \\
Typical length & Few meters \\
Pumping & Optical \\
Gain Spectrum & $\Lambda=1500$ to $1600 \mathrm{~nm}$ \\
Gain Bandwidth & 25 to $35 \mathrm{~nm}$ \\
Relaxation time & 0.1 to $1 \mathrm{~ms}$ \\
Maximum Gain & $30-50 \mathrm{~dB}$ \\
Saturation power & $>10 \mathrm{dBm}$ \\
Crosstalk & nil \\
Polarization & Insensitive \\
Noise figure & $1-4 \mathrm{~dB}$ \\
Insertion loss & $<1 \mathrm{~dB}$ \\
Optics & Pump laser diode couplers, fiber splice \\
Optoelectronic Integration & No \\
\hline
\end{tabular}

\subsection{Applications of the Erbium Doped Silica Fiber Laser}

The main innovation of an $\mathrm{Er}^{3+}$ doped with silica fiber laser is in long distance communication over networks in fiber optics known as "Wavelength Division Multiplexing" (WDM). It is specially applied to 'under water' communication links. Optical signals can be amplified by putting pieces of erbium doped fiber at proper locations in the network [Fig.6] of the fiber.

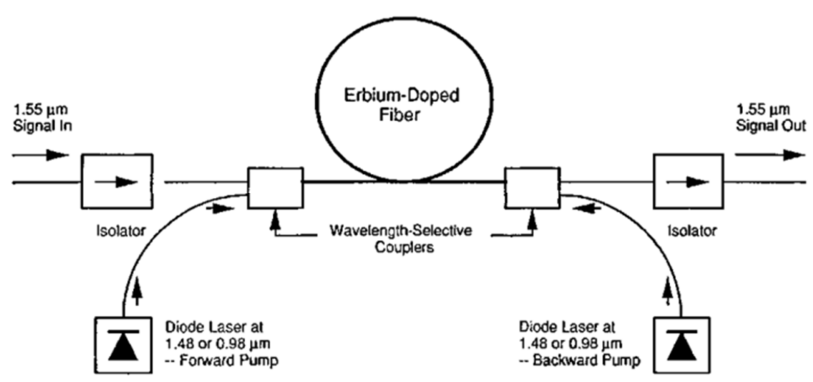

Figure 6. Erbium doped silica fiber as an amplifier. (Courtesy: Wikipedia).

A signal that is optically transmitted then gets an optical boost directly or gets amplified as the signal travels between continents. Since fiber lasers are very easy to construct and operate, they may replace in future, the distributed feedback semiconductor lasers (DFB).Further, the erbium doped fiber lasers have applications in computer links, spacecrafts, process control, industrial automation and other applications. We made a theoretical calculation for an amplifier having a doping concentration of $5 \times 10^{24} \mathrm{~m}^{-3}$, with a signal wavelength $\lambda_{\mathrm{s}}=1.535 \mu \mathrm{m}$ and an absorption cross-section at $\lambda_{\mathrm{s}}, \sigma_{\mathrm{sa}}=4.642 \times 10^{-25} \mathrm{~m}^{2}$, emission cross-section at $\lambda_{\mathrm{s}}, \sigma_{\mathrm{se}}$ $=4.642 \times 10^{-25} \mathrm{~m}^{2}$, with a lifetime for spontaneous emission, $\tau_{\mathrm{sp}}=1.1 \times 10^{-12}$ second. The length of the doped fiber $=10 \mathrm{~m}$ and its confinement factor $\Gamma_{\mathrm{s}}=0.81$. We assumed that it is an erbium doped silica glass fiber and that it has a three-level lasing system and the transitions are characterized by different absorption and emission cross-sections. The erbium ion density is taken as $\mathrm{N}_{\mathrm{Er}}$ and we used a single mode fiber with a pump wavelength of $\lambda_{\mathrm{p}}=0.98 \mu \mathrm{m}$ with a signal wavelength of of $\lambda_{\mathrm{s}}=1.55 \mu \mathrm{m}$. [Ref 8-10]. $\mathrm{N}_{\mathrm{G}}$ is taken as the ground state in $\mathrm{Er}^{3+}$ matrix $\left({ }^{4} \mathrm{I}_{15 / 2}\right)$ and $\mathrm{N}_{\mathrm{M}}$ is taken as a metastable state $\left({ }^{4} \mathrm{I}_{13 / 2}\right)$.Usually, pumping this system by $\lambda$ $=0.98 \mu \mathrm{m}$ will take the ground state erbium ions to the $\left({ }^{4} \mathrm{I}_{11 / 2}\right)$ level also known as excited state level $\mathrm{E}_{2}$.After reaching this level, the ions rapidly undergo relaxation to metastable energy level $\mathrm{E}_{\mathrm{M}}$. Hence.

$$
N_{G}+N_{M}=N_{E r} \text { (erbium ion density). }
$$

We also we assume that $\mathrm{N}_{M} / \mathrm{N}_{\mathrm{Er}}$ is a constant throughout the length of the fiber and is equal to 0.71 , though in practice, it may vary. The total gain $\mathrm{G}$ for a lumped fiber of length $\mathrm{L}$ is now calculated using the equation,

$$
\mathrm{G}=\Gamma_{\mathrm{s}} \exp \left[\sigma_{\mathrm{sa}} \mathrm{N}_{\mathrm{Er}}\left\{\left(\sigma_{\mathrm{se} /} \sigma_{\mathrm{sa}}+1\right) \mathrm{N}_{\mathrm{M}} / \mathrm{N}_{\mathrm{Er}}-1\right\} \mathrm{L}\right] .
$$

The result is gain $\mathrm{G}=30 \mathrm{~dB}$.If we substitute that $\mathrm{N}_{\mathrm{M}} / \mathrm{N}_{\mathrm{Er}}$ $=1$ and $\Gamma_{\mathrm{s}}=1$, then we get maximum possible gain $\mathrm{G}_{\max }=80$ dB.A more realistic value of the $G_{\max }$ can be obtained using the principle of conservation of energy, where the formula for calculation is given by

$$
\mathrm{G}_{\max }=1+\lambda_{\mathrm{p}} / \lambda_{\mathrm{s}}, \mathrm{x}_{\mathrm{p}, \text { in }} / \mathrm{P}_{\mathrm{s}, \text { in }}
$$

where $P_{p, \text { in }}$ is the input pump power at wavelength $\lambda_{p}$ and $P_{s, \text { in }}$ is the pump power at wavelength $\lambda_{\mathrm{s}}$.

\section{Acknowledgements}

The author wishes to acknowledge the constant encouragement given to him by the management and by Prof. Dr. C. V. Jayakumar, Principal, Sri Sairam Engineering College, Chennai to publish research works in reputed international journals.

\section{References}

[1] Digonnet M.J.F., "rare-Earth doped fiber lasers and amplifiers", Marcell Dekker, New York, 2001.

[2] Khare.R.P, "Fiber optics and optoelectronics", Oxford University Press, India.

[3] Mears R.J.L, Reekie I.M, Jauncey and Payne D.N., "Lownoise erbium-doped fiber amplifier operating at $1.54 \mathrm{~mm}$. "Electron Letters" 23, pp1026, 1987.

[4] Amnon Yariv, Pochi Yeh, "Photonics" 6th Edition, Oxford University Press, New York.

[5] Nambiar.K.R, "Laser Principles and Applications", New Age International (P) Limited, Publishers, New Delhi-110002.

[6] Keiji Kuroda, Ayako Suzuki, Yuzo Yoshikuni "Control and probe of population inversion using nanosecond pulse trains in an erbium-doped fiber amplifier", Volume 20, Issue 5, October 2014, Pages 483-486 doi:10.1016/j.yofte.2014.05.014. 
[7] Edgar A. Peralta, Alireza Marandi, and Charles Rudy "Erbium-Doped Fiber Amplifier and Laser", web.stanford.edu/ eperalta/academics/304_Lab5.pdf.

[8] Polman.A, "Erbium as a probe of everything?"Physica B: Condensed Matter, Volume 300, Issues 1-4, 1 July 2001,
Pages 78-90, doi: 10.1016/S0921-4526(01)00573-7.

[9] Desurvire.E,"Erbium doped fiber amplifiers: Principles and Applications", John Wiley \& Sons, New York.

[10] Sze.S.M, "Physics of semiconductor devices", John Wiley, New York, 1981. 